\title{
IT Governance through an Inter-Organizational Workflow based on Multi-Agent System
}

\author{
M.Chergui \\ (EAS- LISER) \\ Systems Architecture Team \\ ENSEM, Hassan II University \\ BP.8118, Oasis Casablanca
}

\author{
A.Sayouti \\ (EAS- LISER) \\ Systems Architecture Team \\ ENSEM, Hassan II University \\ BP.8118, Oasis Casablanca
}

\author{
H.Medromi \\ (EAS- LISER) \\ Systems Architecture Team \\ ENSEM, Hassan II University \\ BP.8118, Oasis Casablanca
}

\begin{abstract}
This work deals with IT governance integration in an information system (IS), since it's one of the main worries to maintain alignment between information technologies and business strategy.

It uses COBIT as IT Governance framework saw its generic, and flexible appearance to produce a measurable picture of the current state of IS, through structured IT control activities.

It appealed to Loose Inter-organizational Workflow, which refers to occasional cooperation, to resolve coordination problems between heterogeneous IS components.

it proposes, afterwards, an organizational model, a collaboration diagram and a generic architecture for the solution.

It gives finally an overview of our implementation limited for the moment to a mediator finding COBIT partners to IS Governance requests.
\end{abstract}

\section{General Terms}

IT Governance, Information system, Multi-agent, System, Inter-organizational Workflow. Agent Group role.

\section{Keywords}

COBIT, Process, Information System, Agent technology, Inter-Organizational Workflow.

\section{INTRODUCTION}

Despite the exponential progress in computer sciences technologies, available to the company (Internet, high-level language, wireless ...), the Information System (IS) does not always provide the expected answers to the top management. Indeed, it is most often found with a phase shift of time, quality of information and obtained results.

This problem is due on the one hand to IS computerization without full compliance with the engineering business rules: problem treated by an investment in IS modeling methods [1].

On the other hand, it's due to the intrinsic complexity and complication of nowadays IS witch make difficult the alignment of Information Technologies with business requirements and strategy of the company: IT Governance matters.

IT Governance describes how an IS is directed and controlled by defining approaches and good principles to implement the search for performance and reducing costs and risks. It can also define the relationship between IS users, the key processes and the common points between technical and functional architectures [2].

Many Governance Risk Compliance (GRC) software are marketed to deal with this problem but the perfect solution should take into account both system and business complexities.

In fact, the most important complexity factors identified in an Information System are: Heterogeity, Autonomy and Evolution.

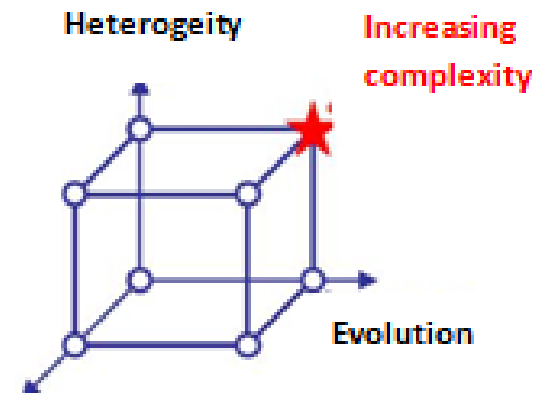

Autonomy

Fig1: Complexity factors

These factors and advanced processes description in organization context had generated the notion of cooperative organizations and Inter-organizational Workflows (IOW).

The contribution of this paper is an IT Governance solution based on COBIT framework and a loose Inter-organizational Workflow modeled by Multi-agent approach.

COBIT is called as a generic IT Governance framework, process-oriented and adaptable to different kind of companies and business.

Loose Inter organizational Workflow to deal with the coordination of IS components for the hole system IT Governance.

The Agent oriented approach to insure components autonomy, and high level communication protocols.

This paper is organized as follows:

Section 2 deals with Information Systems Governance, and presents our problematic. Section 3 recalls inter- 
organizational workflows, cooperative IS and Agent oriented approach. Section 4 describes the modeling of our interorganizational IT GRC Workflow by presenting the organizational model, the generic architecture and the collaboration diagram. Section 5 gives an overview of our implementation. Section 6 presents the eventual perspectives. Section 7 concludes our work.

\section{INFORMATION SYSTEM GOVER-}

\section{NANCE}

\subsection{What is IT Governance?}

IT Governance is a subset discipline of Company Governance focused on information technologies and communication. This discipline, in line with business objectives, focuses on risk management, optimization of investments and resources, and value creation. It is indeed, a balance between governance and information technology management in within the company [3].

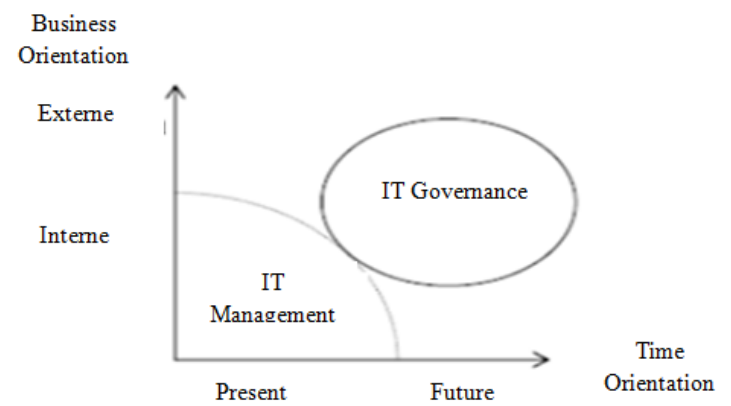

Fig2: Positioning IT Management over IT Governance.

The difference between these two components helps to provide a better overview of what the IT Governance is. The IT Governance provides guidance and control management processes within the company and this via:

-The strategic direction of the management process, -The operating business processes to provide the services requested,

-The control of smooth processes, their improvements and defining new directions.

\subsection{Frameworks and software solutions}

\subsubsection{Frameworks}

To implement and achieve optimal companies IT governance, many standards or normative principles have been defined in recent years; from the control frameworks such as COBIT and Val IT to the best practices for implementation, such as ITIL and CMMI. Each follows a specific function.

The French Association of Normalization (AFNOR) has a house presentation of these frameworks to be applied within Information System Management (ISM):

- In foundation of the house, the quality assurance methods (ISO 9001).

-On the ground floor, transverse methods of project management (PMP in the scheme, but it can also be Prince2).
- Upstairs, operational methods for the two major pillars of ISM which are Development (CMMI method) and production (ITIL, ISO 20000)

- At the heart of the house, a method focused on security (ISO 27001).

- At roof level, generic methods of governance SI (COBIT), as the strategic level

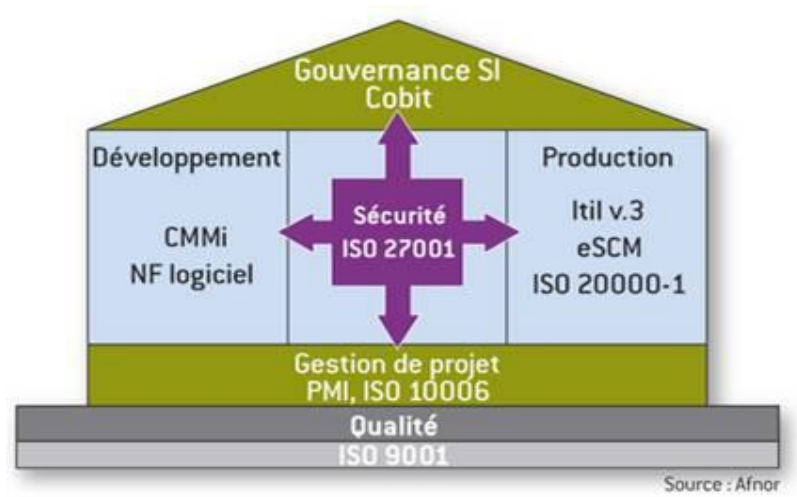

Fig3: Information System methods house by AFNOR

In this solution, COBIT V4.1 was chosen as IT governance framework for its generic aspects; COBIT can also include other standard and give recommendations for the best IT governance practices for the company without focusing only on security, project management or services production.

\subsubsection{COBIT V4.1}

Control Objectives for Information and related Technology (COBIT) provides good practices across a domain and process framework and presents activities in a manageable and logical structure. COBIT's good practices represent the consensus of experts. They are strongly focused more on control, less on execution [4].

COBIT control framework contributes to establish an internal control system in by:

$\Rightarrow$ Making a link to the business requirements

$\Rightarrow$ Organizing IT activities into a generally accepted process model

$\Rightarrow$ Identifying the major IT resources to be leveraged

$\Rightarrow$ Defining the management control objectives to be considered.

COBIT defines IT activities in a generic process model within four domains, namely:

Plan \& Organize, Acquire \& Implement, Deliver \&Support, and Monitor \& Evaluate.

These domains contain 34 generic processes, managing the IT resources to deliver information to the business according to business and governance requirements.

COBIT offers many components interrelated, to provide support for the governance, management, control and assurance needs of the different audiences as shown in the figure bellow: 


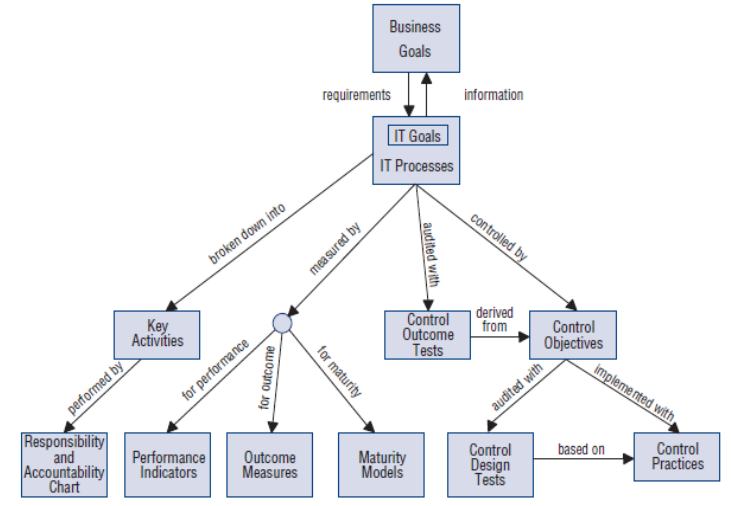

Fig4: Interrelationship between COBIT Components

In what follows, this components relationship will be used to define COBIT Agent's architecture and COBIT audit approach to define the processes of IT Governance Workflow.

\subsubsection{IT GRC Software}

Many leader software editors propose IT GRC solutions to support companies' worries about their IS governance. In fact, as for the Gartner [5], In December 2011, the IT GRC solutions most common on the market are:

Table 1: IT GRC vendors and products' information

\begin{tabular}{|c|c|c|c|}
\hline Editor & Solution name & $\begin{array}{l}\text { Product } \\
\text { version }\end{array}$ & $\begin{array}{c}\text { Version } \\
\text { release date }\end{array}$ \\
\hline Agiliance & $\begin{array}{l}\text { Agiliance } \\
\text { RiskVision }\end{array}$ & V 6.0 & May 2011 \\
\hline ANXeBusiness & ControlCase GRC & V4.0 & $\begin{array}{l}\text { August } \\
2010\end{array}$ \\
\hline ControlCase & TruComply & V6 & $\begin{array}{l}\text { December } \\
2010\end{array}$ \\
\hline Easy2Comply & Easy2Comply & V4.7.5 & June 2011 \\
\hline Modulo & $\begin{array}{l}\text { Modulo Risk } \\
\text { Manager NG }\end{array}$ & V7.2 & May 2011 \\
\hline RSA Archer & $\begin{array}{l}\text { RSA Archer } \\
\text { eGRC Platform }\end{array}$ & V5.0.6 & $\begin{array}{l}\text { December } \\
2010\end{array}$ \\
\hline Rsam & Rsam & V7.2 & $\begin{array}{l}\text { October } \\
2010\end{array}$ \\
\hline Symantec & $\begin{array}{l}\text { Symantec Control } \\
\text { Compliance Suite } \\
\text { (CCS) }\end{array}$ & V10.5 & $\begin{array}{l}\text { December } \\
2010\end{array}$ \\
\hline
\end{tabular}

Through these solutions performance and strengths comparison [5] we notice that:

-All of them are GRC solution for company business (financial services, statistic analysis...), and not Information system governance tools,

-Most of them are part of an ERP and need other business modules to be operational (high cost of implementation).

-Most of them are not adapted to small and medium companies IS.

-All of them don't focus on IS Management best practices but on business ones.

\subsection{Problematic}

Despite the existence of many GRC software, there is a need of a complete IS Governance solution which is based on best practices and adaptable to the company nature and context. Moreover, IS governance solution should be permanently active to support and evaluate the system with the effective participation of its own users.

It's in this perspective that this work was done, the idea is to design a workflow based solution able to be integrated in any kind of IS and based on COBIT practical audit approach namely:

$\checkmark$ Choose the interviewee

$\checkmark$ Ask questions based on the objectives of process control

$\checkmark$ Choose the process

$\checkmark$ Rate responses

$\checkmark$ Decide priorities,

$\checkmark$ Provide measures and indicators.

This Workflow has intelligent components ready to translate permanently users governance worries about the part of the IS they use.

\section{WORKFLOW AND INFORMATION SYSTEM COORDINATION}

\subsection{Cooperative Information System and Inter-Organizational Workflows}

Cooperative Information system (CIS) is a new generation of IS, born to ensure the cooperation in different levels between many IS in a complex communication network. CIS is defined as a group of preexistent autonomous components that work in synergy to exchange information, expertise and to coordinate activities [6].

CIS implement the notion of Workflow to ensure the automatic management of information flows. A Workflow is an automation of a business process that defines a set of component tasks, their coordination, the information and performers involved in each task.[7].

Inter-Organizational Workflow (IOW for short) is an extension of traditional workflow. It's a composition of several workflows that support cooperation between distributed and heterogeneous business processes running in different organizations with common resources and skills to reach a common goal [8]. 
IOW can be investigate in two scenarios [9] :

- Loose IOW: occasional and opportunist cooperation, without structural constraints, where the partners involved and their number are not pre-defined,

-Tight IWO: structural cooperation among organizations with a well-established infrastructure among pre-defined partners. Involved organizations, in this case are engaged in a longterm cooperation and their workflows (business processes) are interdependent.

In this article, the Loose IOW is chosen since we deal with IS components which are heterogeneous and not obligatory interdependent.

An inter-organizational workflow processes two kinds of problems:

$\checkmark$ The local problem concerns each Workflow Management System (WFMS) of the IOW,

$\checkmark$ The global problem concerns the global WFMS.

The IOW raised several constraints under the local and global problems namely:

$\checkmark$ Heterogeneity of WFMS development platforms

$\checkmark$ Autonomy of each organization participating in the IOW as far as conditions of cooperation are concerned.

$\checkmark \quad$ Flexibility of tasks allocation in partners' selection

$\checkmark$ Distribution of implemented processes, in formations and resources.

It's the raison why Agent-Oriented approach was chosen to model IT Governance IWO since this technology satisfies almost all these constraints: autonomy, flexibility and distribution.

\subsection{Multi-agent System}

There is no unified definition of an Agent but the closest to our vision is: An agent is an autonomous real or abstract entity that is capable of acting on itself and its environment, which, in a multi-agent world, can communicate with other agents, and whose behavior is the result of observations, knowledge and interactions with other agents [10].

In this case, not only one agent is used but a set of agents witch interact among each other that are called Multi-agent system (MAS).

\section{An MAS is characterized by:}

- Every agent in the system has his own knowledge and way to resolve problems.

- There is no global control of an MAS,

- The Data in MAS is decentralized.

\subsection{Agent Group Role}

Agent-Group-Role (AGR) is a Meta model of MAS organization.

This model considers the agent as an active communicating entity that can play one or more roles through membership in a group or groups without any constraints on its architecture [11].

$\Rightarrow$ A group is a set of agents with common characteristics, used as a business model. Two agents can communicate only if they belong to the same group.

$\Rightarrow$ A role is an abstract representation of the activity of an agent in a group, it can be played by several agents, it is specific to each group and it is requested by the agent who wants to play it.

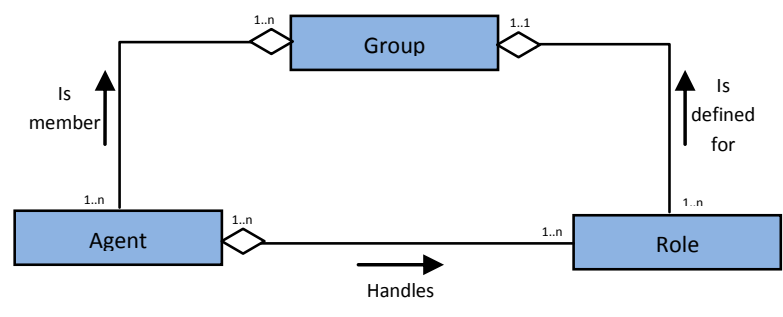

Fig 5: UML Diagram of AGR model.

It's the model adopted in this work since it's adaptable to a loose IOW context by providing the appropriate concepts to describe the macro level of agents cooperation.

\subsection{Multi-Agents System Communication}

MAS Communication is a particular form of action that affects the mental representations of agents to make changes in the environment. It must also be modeled as an act that could affect the status of other agents [12].

There are two kinds of Communication procedures:

$\Rightarrow$ Information sharing: it is historically the first model, where shared memory is seen as a table on which agents write and find partial answers and information.

$\Rightarrow$ Sending messages: proposed by Hewitt [13] actor model. Defining an actor as an active and autonomous entity that has a partial view of the universe. This actor is characterized by: acquaintances and behavior described by a script (set of methods that indicate the various actions that can accomplish this actor in response to the messages it receives).

In this case we use :

-sharing information way for the communication between IS workflow Agent and IS Connection Server and between Cobit Agent and Cobit Connection Server.

-Sending messages way for the communication between Connection Server Agents and the mediator Agent and between Cobit Agent and IS Workflow Agent to negotiate the audit contract.

\section{IT GOVERNANCE WORKFLOW MODELING}

As a solution of the problematic, this article proposes an architecture and an organizational model of a Loose Interorganizational Workflow based on COBIT framework (4.1 version). It's a solution purely dedicated to the information system governance. It uses the practical approach of COBIT to audit permanently every component of the Information System, and to be sure of its alignment with the company business objectives.

The vision is that the IT Governance dimension should be omnipresent in the daily functioning of IS with the participation of its potential users.

COBIT was chosen as it's a generic IT Governance framework (any kind and size of companies), able to audit the 
IS, to measure its actual state and to give the global directives to improve it. In add, the process oriented approach of COBIT provides an intelligent dimension useful for an automated audit.

IOW was used to solve the problem of Heterogeity of nowadays IS components, their space distribution and their scalability.

The proposed solution opted afterwards for the Loose IOW to subdivide any IS Governance to the IT Governance of any software in it. Software are not obligatory neither known in advance nor in communication between them.

Multi-agent system was called to offer many advantages sought in this kind of workflow namely:

$\Rightarrow$ Intelligence
$\Rightarrow$ Autonomy
$\Rightarrow$ Distribution
$\Rightarrow$ High level communication protocols
$\Rightarrow$ Entity learning

\subsection{Organizational model}

The proposed organizational model is based on Agent-GroupRole Meta-model; it defines the macro-level dimension of our Inter-organization workflow organization, independently of the internal features of each participating component.

Our organizational model (see Fig6) is organized around the following components:

- Five types of groups represented by an eclipse (Audit, Finding Audit, Finding Auditor, Audited and Auditor)

- Ten roles represented by a circle as every agent has double role in every group ( Mediator, SI Connection Server, COBIT Connection Server, IS Workflow Agent, COBIT Agent)

- Communication between agents is represented by arrows.

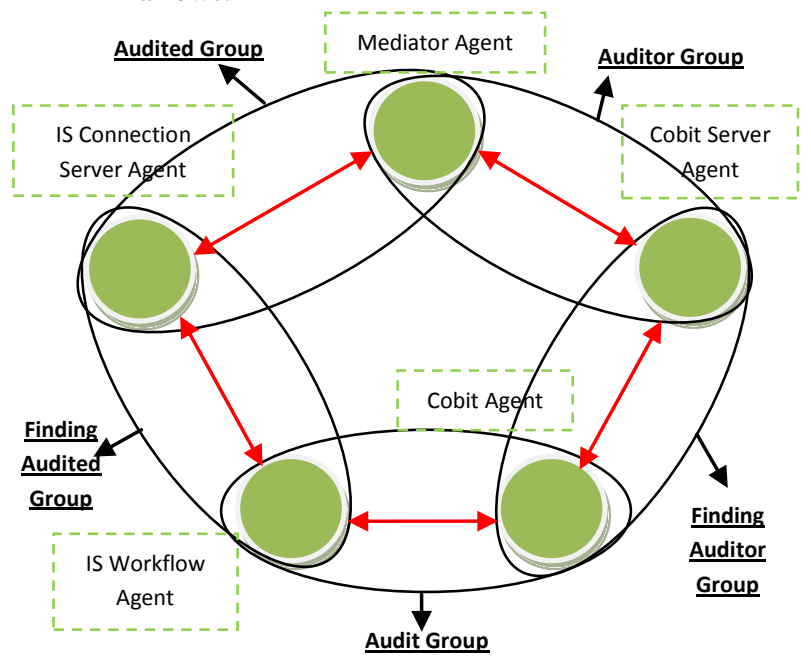

: Role
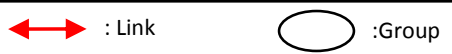

Finding Audited Group and Finding Auditor Group allow one or more IS Workflow Agent or COBIT Agent to interact with a connection server (COBIT or IS) from which they get requested partner identity.

Auditor Group allows connection server via a mediator Agent (recording COBIT Agents capabilities), to release the appropriate COBIT process (offered by a COBIT Agent).

Audited Group allows the connection server to submit an audit request about a business objective; it allows the mediator agent to return the identity of the appropriate COBIT agent.

Audit Group created by the IS Workflow Agent and COBIT Agent, after getting each other identities from connection servers. It allows them to negotiate the more priority COBIT process to implement; the RACI matrix, the key metrics and the maturity model to follow. This is the audit procedure.

\subsection{Architecture}

The overall architecture is based on:

- The agentifacation of COBIT 4.1 components relationship - The reference architecture of Loose IOW

In fact, the IT Governance IOW has a direct interface with every part of the IS throw an Agent which is started by users requests about the audit of one or many processes of the system they use. This Agent is called IS Workflow Agent.

IS Workflow Agent represents a business objective born from pertinent remarks noted in the use of the application or in functional meeting about it. It concerns most often the control of the software alignment to the company vision about risk, investment, optimization ...etc.

Manager Agent manages and controls IS Workflow Agents through:

$\checkmark \quad$ Creating or updating new IS Workflow Agent

$\checkmark$ Sharing resources and their coordination

$\checkmark$ Suspension or termination of IS Workflow Agents

$\checkmark$ Persistence.

Fig5: Organizational Model of the IT Governance IOW based on COBIT. 


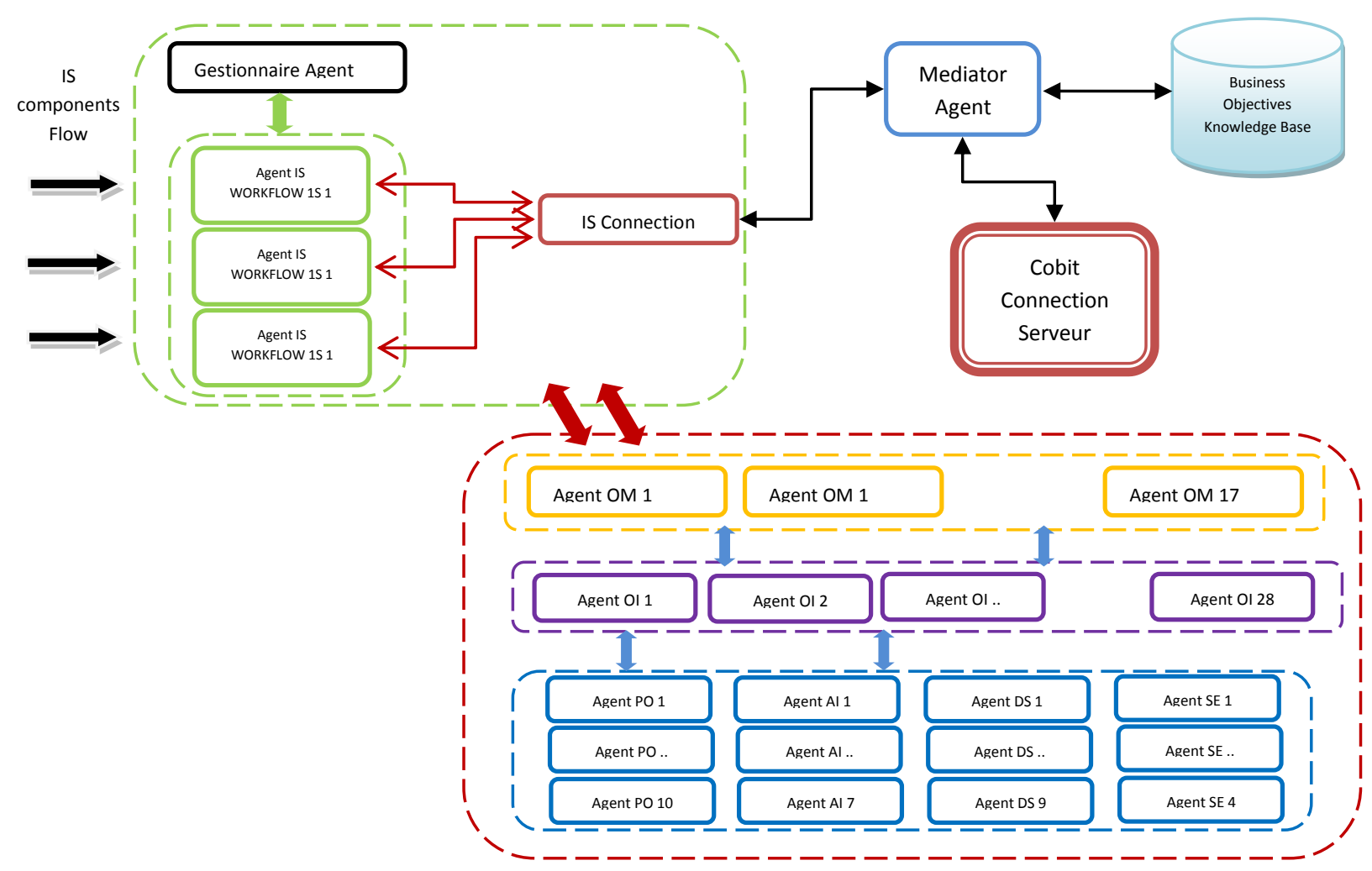

Fig 6: Generic architecture of It Governance Loose IOW based on COBIT V4.1

COBIT Agent: it's the auditor agent. It broadcast its services throw the COBIT Connection Server. Once into contact with an IS Workflow Agent, COBIT Agent calls other agents it manages, namely:

\section{$>$ IT Objective Agents \\ $>$ Process Agents}

They diagnose the business objective coming from IS Workflow Agent and give convenient recommendations as it's done in COBIT framework.

Connection Server Agent: it's the agent which is responsible for publishing Workflow IS Agents requests to the mediator agent and getting convenient COBIT Agents identity also from the mediator agent.

Mediator Agent: it's a yellow pages service that publishes COBIT Agents offered services on the one hand and secondly to publish requests made by the IS Workflow agents in the other hand. This is to match the best offer for an audit requests so as to establish the connection between the two parts.

\section{IMPLEMENTATION OVERVIEW}

So as to validate our generic architecture, a simulator of our IT Governance workflow is developed. Madkit 5 platform is used as it natively integrates the AGR model, Eclipse as integrated development environment and java as development language.
Here is the collaboration diagram of the simulator presenting different kind of agents and their interaction:

the Agents framed in red are finalized and; namely:

Mediator Agent, IS Connection Server and COBIT Connection Server

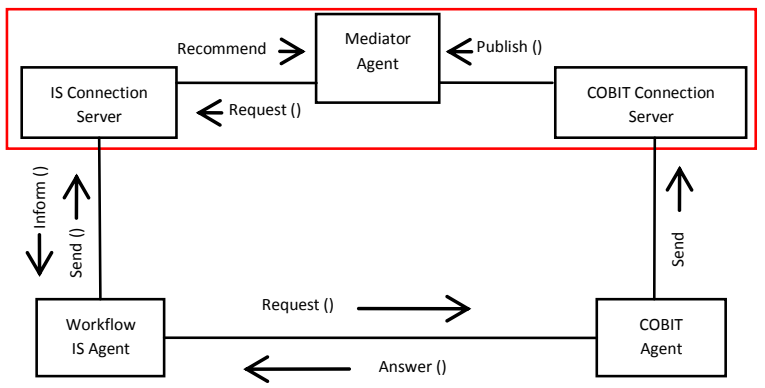

Fig 7: Collaboration Diagram of the simulator

We present below our simulator with the 3 different Agent Roles 


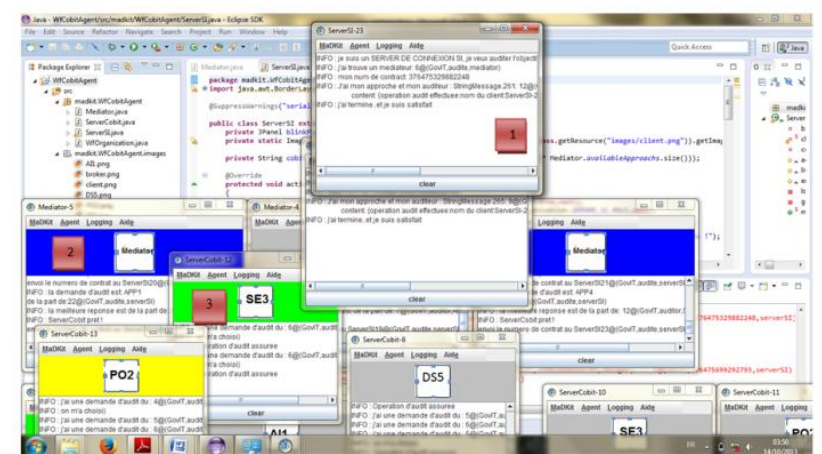

Fig 8: Overview of IT Governance IOW

It's supposed that an IS Connection Server has a business objective to which he will find the convenient COBIT Agent to audit it (in our case we will stop in the level of exchanging the audit contract number between the two connection servers through the Mediator which establish the correspondence).

To simulate this operation, a graphic interface was used for every agent, the request is imitated to find the convenient COBIT connection server publishing one of COBIT processes (the choice is based on the mediator matching)

Interface 1: IS connection Server Agent named ServerSI+ excremental number. This agent presents a business objective, it asks the mediator to find an auditor and wait for an answer.

Interface 3: COBIT Connection Server named ServerCobit+ excremental number. This agent publishes its service through the mediator, and waits to be chosen as an auditor.

Interface 2: Mediator Agent matches the audited agent with the convenient auditor agent. It sends the answer to the Connection servers.

\section{PERSPECTIVES}

As perspective of this work, a communication language and ontolgies will be defined in each part of the Workflow; the knowledge base of the mediator and detailed the architecture of different agents will be proposed.

\section{CONCLUSION}

This article proposed an architecture of Inter-Organization Workflow of IT Governance based on COBIT 4.1 framework.

In fact it presented first IT GRC, its frameworks and the problematic of its solutions.

It briefly talked after that, about cooperative Information system, Workflows and Agent technology. Then it presented the organizational model and the architecture of the solution, validated with a simulation developed on Madkit 5 plateform.

The message of this paper is that organizational perspective has an important role in the company IT Governance. The use of an inter-organizational Workflow with an intelligent dimension (Multi-Agent System) allows solving permanently the problem of Information System alignment with Company Business objective.

\section{REFERENCES}

[1] Barbier, F., Cauvet, C., Oussalah, M., Rieu, D., Bennasri, S., \& Souveyet, C. (2002). Composants dans l'ingénierie des systèmes d'information: concepts clés et techniques de réutilisation. Actes des deuxièmes assises nationales $d u$ GDR-I3.

[2] Gary Hardy Using IT governance and COBIT to deliver value with IT and respond to legal, regulatory and compliance challenges information security technical report11 2006 page 55-61

[3] De Haes, S., \& Van Grembergen, W. (2004). IT governance and its mechanisms. Information Systems Control Journal, 1, 27-33.SIGCHI Conference on Human Factors in Computing Systems

[4] COBIT 4.1.- http://www.isaca.org

[5] C. McClean The Forrester Wave ${ }^{\mathrm{TM}}$ : IT Governance, Risk, And Compliance Platforms, Q4 2011 Report December 2011.

[6] De Michelis G.,Dubois E.,Jarke M.,Matthers F., Mylopoulos J.,Papazoglou M .,Polh K.,Schmidt J.,Woo C.,Yu E. - Cooperative information systems: A manifesto, Cooperative Information System : Trends and Directions, (1997).

[7] S.Nurcan. Analyse et conception de systèmes d'information coopératifs. Numéro thématique "Multimédia et collecticiel" de TSI (Techniques et Science Informatiques), Vol. 15, $\mathrm{n}^{\circ}$ 9, 1996, p. 12871315.

[8] Aubert, B. A., \& Dussart, A. (2002). Systèmes d'information inter-organisationnels. CIRANO.

[9] M. Divitini, C. Hanachi, C. Sibertin-Blanc, "Inter organizational Workflows for Enterprise Coordination". chapter 15 of Coordination of Internet Agents. In A. Omicini, F. Zambonelli, M. Klusch, R. Tolksdorf, editors, Springer, Berlin, Germany 2001.

[10] : A. SAYOUTI, H. MEDROMI -"Les Systèmes MultiAgents : Application au Contrôle sur Internet" Auteurs Éditions universitaires européennes, Août 2012.].

[11] Hübner, J., Sichman, J. S., \& Boissier, O. (2002). Spécification structurelle, fonctionnelle et déontique d'organisations dans les systemes multiagents.JFIADSMA, 2, 205-216.

[12] A.SAYOUTI \& H. MEDROMI Autonomous and Intelligent Mobile Systems based on Multi-agent, Book Chapter in the book " Multi-agent Systems - Modeling Control , Programming, Simulations and Applications”

[13] Sayouti, A., Qrichi Aniba, F., \& Madromi, H. (2008, November). Interactions between agents as shared resources in multi-agents systems. In New Technologies, Mobility and Security, 2008. NTMS'08. (pp. 1-4). IEEE. 